\title{
Numerical Analysis and Design of Photonic Crystal Fiber Based Surface Plasmon Resonance Biosensor
}

\author{
Md. Biplob Hossain'1, Md. Sanwar Hossain'2, Md. Moznuzzaman1, Md. Amzad Hossain', \\ Md. Tariquzzaman', Md. Tanvir Hasan'1, Md. Masud Rana ${ }^{3}$
}

\begin{abstract}
${ }^{1}$ Department of Electrical and Electronic Engineering, Jashore University of Science and Technology, Jashore, Bangladesh ${ }^{2}$ Department of Electrical and Electronic Engineering, Bangladesh University of Business and Technology, Dhaka, Bangladesh ${ }^{3}$ Department of Electrical and Electronic Engineering, Rajshahi University of Engineering \& Technology, Rajshahi, Bangladesh Email: biplobh.ee10@gmail.com, sanwareee@gmail.com,m.moznuzzaman@just.edu.bd, mahossain.eee@gmail.com, swapnilta riq231@gmail.com, mth@just.edu.bd, md.masud.rana.ruet@gmail.com
\end{abstract}

How to cite this paper: Hossain, Md.B., Hossain, Md.S., Moznuzzaman, Md., Hossain, Md.A., Tariquzzaman, Md., Hasan, Md.T. and Rana, Md.M. (2019) Numerical Analysis and Design of Photonic Crystal Fiber Based Surface Plasmon Resonance Biosensor. Journal of Sensor Technology, 9 , 27-34.

https://doi.org/10.4236/jst.2019.92003

Received: June 1, 2019

Accepted: June 27, 2019

Published: June 30, 2019

Copyright () 2019 by author(s) and Scientific Research Publishing Inc. This work is licensed under the Creative Commons Attribution International License (CC BY 4.0).

http://creativecommons.org/licenses/by/4.0/

\begin{abstract}
In this paper, a high sensitive photonic crystal fiber (PCF) based surface plasmon resonance (SPR) biosensor is numerically studied. In this structure, as a plasmonic material, gold $(\mathrm{Au})$ is used because of its chemical activeness. And a layer of sensing medium is used outside of the fiber to make the structure effective. Any unknown biomolecular analyte can be detected by placing or flowing it on the metal surface. Guiding properties and results are investigated using Finite element method (FEM). Results show that maximum sensitivity is $4000 \mathrm{~nm} / \mathrm{RIU}$, as well as resolution, is $2.5 \times 10^{-5} \mathrm{RIU}$ of the proposed sensor.
\end{abstract}

\section{Keywords}

Biosensor, PCF, SPR, SPP Mode, Sensitivity

\section{Introduction}

The surface plasmon is referred as the plasma oscillation which is localized at the surface or interface. "Plasmon" means quasi-particle representation of plasma frequency [1]. Plasma oscillation represents the phenomena that free electrons of the metal oscillate cooperatively from their equilibrium position [2]-[7]. In SPR measurement the change of refractive index change in nanometer range can be detected easily. For this reason SPR is used in different bio-sensing application such as detection of label-free antigen-antibody reaction.

In the last couple of years, SPR biosensor has been created a remarkable place 
from the other biosensors because of its high sensitivity and reliable procedure [8]. Kretschmann configuration [8] is widely used in which a very thin layer of metal is directly deposited on the surface of coupling prism. It is bulky in size and difficult to optimize. Furthermore, it cannot be used in remote sensing application. For removing this barrier of remote sensing optical fiber is used. Its advantage is that its core diameter is small that is why it can be used in small areas along with the capability of remote sensing. In the last decade, PCF has been contributed to different applications owing to its several properties [9] and several types of sensor have been designed using PCF. The PCF SPR sensor is worked on evanescent field principle and it is produced because of propagation of light in the core-cladding region [10]. Owing to changing of analyte local refractive index (RI), the RI of the surface plasmon polaritons (SPP) will be changed and finally, resonant wavelength will be also changed [10].

R.C. Jorgenson has propounded in [11], the very first optical fiber SPR sensor in where the core was coated by the Au film to exhibit plasmonic response. In [12], Polymer selectively coated PCF structure has been put forth by J. N. Das and R. Jha. Moreover, in [13] J. N. Das and R. Jha used multiple graphene layers in birefringence control PCF structure for SPR sensor. Previously the multiple metal layers are exercised in PCF SPR sensor and liquid infiltration inside the air holes or core [14]. Recently PCF SPR biosensor is designed by keeping the metal as well as sensing absorbate outside the PCF structure [15] [16].

Comprehensively as plasmonic material, gold and silver have been used. Among them, silver is preferable due to its most conductive nature, sharp peak and high detection accuracy compared to gold [17]-[25] But silver can be easily oxidized and detection accuracy will be reduced. Silver oxidization can be prevented by using a thin layer of graphene on the silver layer [26] [27] [28]. But gold is chemically immutable and shows larger wavelength shift in resonant wavelength. As a result detection accuracy to detect unknown analyte is raised [28] [29] [30] [31] [32].

In this work, a regular hexagonal PCF SPR biosensor has been proposed where the metal (gold), as well as sensing absorbate, is placed outside the PCF structure. This reduces the fiber structure complexity and sensing process easier. The unknown analyte can be easily detected by flowing it or just placing it outside of the gold surface just in label-free detection mode. Our structure is easy to construct in terms of fabrication.

\section{Design Methodology}

At the outer circle, six circles are omitted so that evanescent field can be flown to excite the metal surface. The cross section of our numerically proposed sensor has been shown in Figure 1. A simple three-ring, hexagonal PCF is designed for the SPR sensor.

According to the standard fiber drawing "stack and draw" technique, the proposed fiber can be constructed. In our core guided mode, the air holes in the 


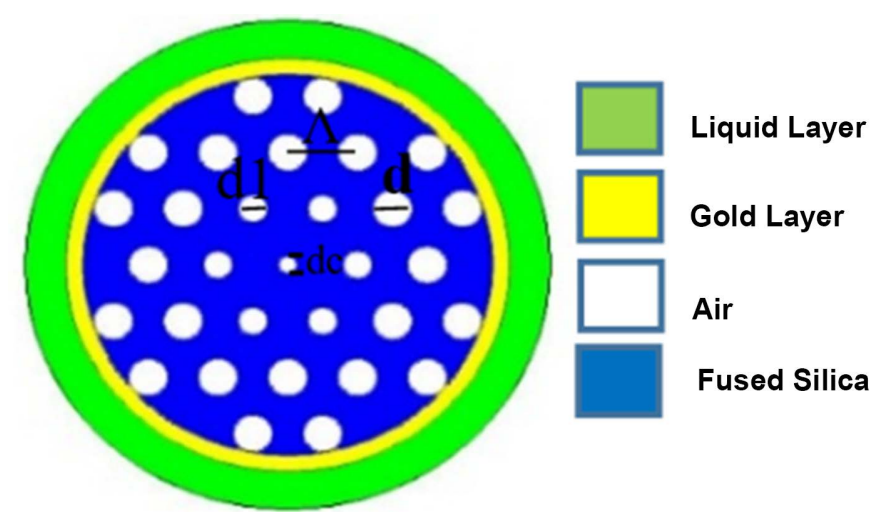

Figure 1. Cross Section view of propounded Sensor.

first ring act as low index cladding. It has been used to vacillate the phase matching with plasmonic mode by reducing the refractive index. Our sensor parameters are defined as pitch $\Lambda=1.7 \mu \mathrm{m}$, diameter of the first ring air hole (d1), the second air hole (d2) and the diameter of the centre air hole (dc) are $0.4 \Lambda$, $0.55 \Lambda$ and $0.25 \Lambda$ respectively. Moreover, the thickness of the gold layer is defined to $40 \mathrm{~nm}$ and it is about the ideal case. The real part of the dielectric constant of the gold has been defined from the Drude-Lorentz model [16]. The RI of silica has been taken by using Sellmeir equation [16].

$$
n^{2}(\lambda)=1+\frac{B_{1} \lambda^{2}}{\lambda^{2}-C_{1}}-\frac{B_{2} \lambda^{2}}{\lambda^{2}-C_{2}}-\frac{B_{3} \lambda^{2}}{\lambda^{2}-C_{3}}
$$

where $B_{1}=0.69616300, B_{2}=0.407942600, B_{3}=0.897479400, C_{1}=4.67914826 \times$ $10^{-3} \mu \mathrm{m}^{2}, C_{2}=1.35120631 \times 10^{-2} \mu \mathrm{m}^{2}, C_{3}=97.9340025 \mu \mathrm{m}^{2}$ are the coefficients of Sellmier equation [16] and $\mathrm{n}$ is the RI of the silica and $\lambda$ is the wavelength in $\mu \mathrm{m}$. We use finite-element method (FEM) and also use scattering boundary condition (SBC) on the outest layer to determine mode confinement behavior.

\section{Results and Discussion}

The working fashion of the PCF based on SPR sensor is evanescent electromagnetics field. For plasmonic phenomenon efficient excitation is the key factor. At a definite frequency of the incident light which can excite the surface and produce resonance, The Finite Element Method (FEM) based software such as "Comsol Multiphysics 4.4" framework has been used to simulate and analyze our proposed structure. Although there exists both the horizontal and vertical polarization, we consider only horizontal polarization here because vertical polarization is lesser compared to the horizontal polarization.

Figure 2 shows, the horizontal polarization property of fundamental core mode and surface plasmon mode.

As the mode propagates through the core, plasmon mode at the metal sensing layer is excited by the core mode. Resonance occurs when the real part of the local RI of the core mode and the surface plasmon mode matches. At this specific point maximum modal loss is found. At maximum loss point imaginary value of 


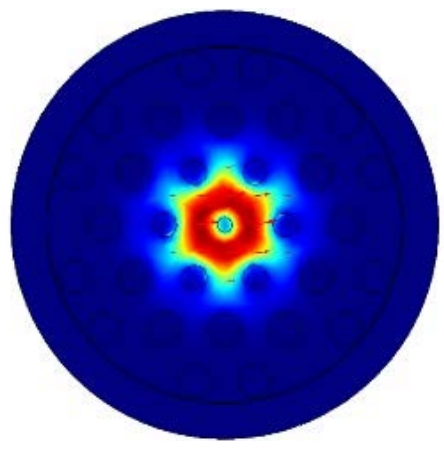

(a)

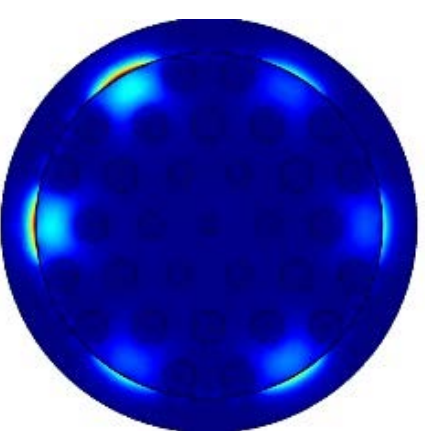

(b)

Figure 2. Field properties of (a) core guided mode for x-polarization and (b) Surface Plasmon Polarition mode.

RI of the core guided mode reaches to largest value. After this point, it has been noticed, modal loss is started decreasing again between core mode and plasmon mode. The modal loss of the fundamental core guided mode is extracted by using the equation below [19].

$$
\alpha=8.686 \times k_{0} \cdot \operatorname{Im}\left[n_{\text {eff }}\right] \mathrm{dB} / \mathrm{m}
$$

where $k_{-} 0=2 \pi \lambda, \lambda$ are the wave number, wavelength in $\mu \mathrm{m}$ in free space.

Figure 3 shows that at $640 \mathrm{~nm}$ wavelength a very sharp peak is found. At that point real part of the core mode and SPP mode coincide. It defines that maximum power has transferred to fundamental core mode to SPP mode. Due to core cladding refractive index contrast sharp peak is found. Without influenced by other higher-order plasmonics one resonance is found for the fundamental mode. Sensing performance is indicated by the presence of resonance on the metal surface. At wavelength $640 \mathrm{~nm}$ phase matching phenomena is found where fundamental core mode, SPP mode and loss peak are well matched.

Our sensor is providing very sensitive results according to the analyte RI change. RI changing will change the phase matching phenomena between the core mode and SPP mode. RI change can be detected by measuring the loss spectra shift. By increasing the analyte RI loss spectra is shifted toward the higher wavelength. It is shown in Figure 4.

\subsection{Wavelength Interrogation Technique}

The advantage of Wavelength Interrogation technique is that it has higher sensitivity as well as higher detection range compared to the Amplitude detection technique [10]. In this method, RI variation is detected by measuring the resonance shift $\Delta \lambda_{\text {peak }}$. Sensitivity in this method is defined as [20].

$$
S_{\lambda}\left[\frac{n m}{R I U}\right]=\Delta \lambda_{\text {peak }} / \Delta n_{a}
$$

where $n_{a}$ is the analyte refractive index. In this method, positive RI sensitivity is found $4000 \mathrm{~nm} / \mathrm{RU}$ which is 4 times higher than the ref. [10] and 2 times than [16]. For the analyte RI, 1.34, 1.35, 1.36 and 1.37 resonant peak is found at 640 , 


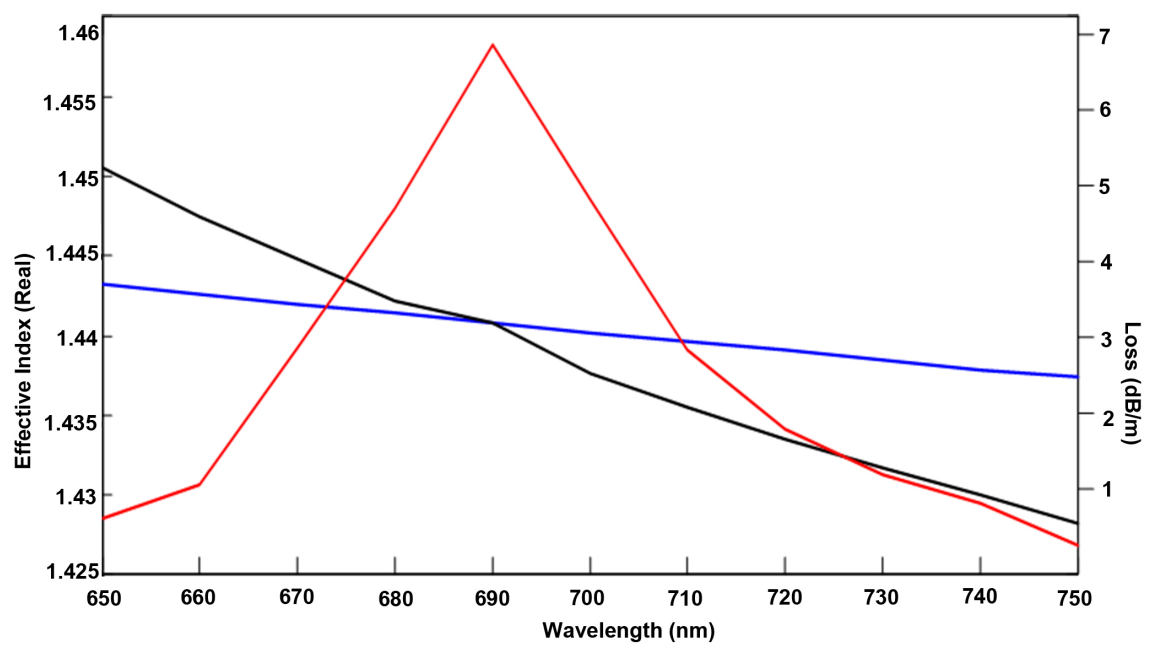

Figure 3. Relation of dispersion of core mode and surface plasmon polarition mode.

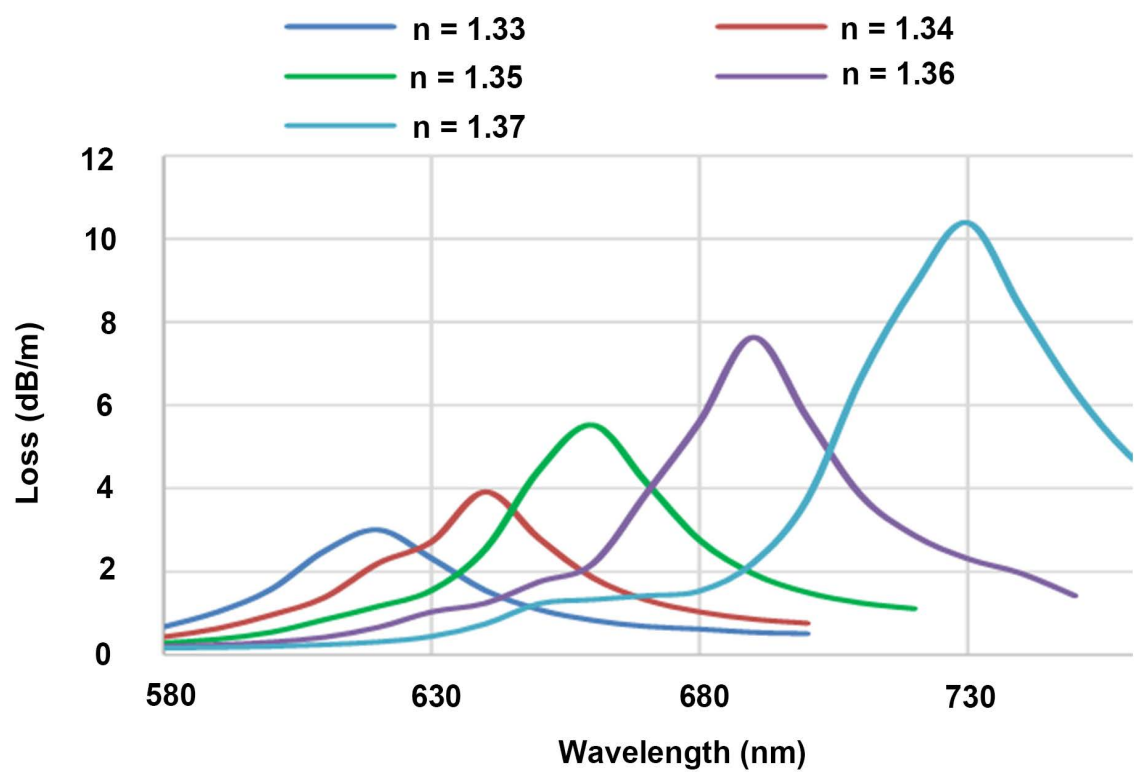

Figure 4. Loss spectrum profile of our proposed sensor by changing analyte RI from 1.33 to 1.3 .

660, 690 and $730 \mathrm{~nm}$ and corresponding sensitivity is found as 2000, 2000, 3000 and $4000 \mathrm{~nm} / \mathrm{RIU}$ respectively. Only higher sensitivity does not mean that the sensor can detect analyte accurately. By measuring sensor resolution it is possible to find that the sensor can detect analyte accurately or not. If we presume the proposed sensor can detect 0.1 spectral resolution then the sensor resolution is $2.5 \times 10^{-5} \mathrm{RIU}$ which is also better than in [10].

\subsection{Amplitude Interrogation Technique}

The advantage of this method is that this method is simple and cost effective because no spectral manipulation is needed here. Sensitivity in this method can be derived as in [10]. 


$$
S_{A}(\lambda)\left[\mathrm{RIU}^{-1}\right]=-\frac{1}{\alpha\left(\lambda, n_{a}\right)} \frac{\delta \alpha\left(\lambda, n_{a}\right)}{\delta n_{a}}
$$

Here, the overall propagation loss is denoted by $\alpha\left(\lambda, n_{a}\right)$ along the wavelength at a specific analyte RI as well as the difference between the loss spectra before and after the analyte RI change is denoted $\delta \alpha\left(\lambda, n_{a}\right)$. By using this method maximum $265 \mathrm{RIU}^{-1}$ is found at $730 \mathrm{~nm}$ wavelength for the analyte RI of 1.37. Moreover, amplitude sensitivities are found 81,89 and $153 \mathrm{RIU}^{-1}$ are found for $1.34,1.35$ and 1.36 RI. If we assume, $1 \%$ change in transmitted intensity can be sensed accurately then the sensor resolution is found $3.7 \times 10^{-5}$ RIU.

\section{Conclusion}

A numerically PCF based SPR biosensor has been proposed and studied. The FEM method has been adopted to model the computational domain of the proposed sensor and a circular perfectly matched layer has been used outside the structure to abolish radiation toward the surface. The proposed sensor shows wavelength sensitivity of $4000 \mathrm{~nm} / \mathrm{RIU}$ with resolution of $2.5 \times 10^{-5} \mathrm{RIU}$. Also, the sensor shows the amplitude sensitivity of $265 \mathrm{RIU}^{-1}$ with resolution of $3.7 \times$ $10^{-5}$ RIU. As the sensor is easy to fabricate by using draw and Stack method. Due to promising result and simple sensing scheme we believe that this sensor can be a good candidate for biological and biochemical analyte detection.

\section{Conflicts of Interest}

The authors declare no conflicts of interest regarding the publication of this paper.

\section{References}

[1] Yamamoto, M. (2002) Surface Plasmon Resonance (SPR) Theory: Tutorial. Review of Polarography, 48, 209-237.

[2] Shushama, K.N., Rana, M.M., Inum, R. and Hossain, M.B. (2017) Graphene Coated Fiber Optic Surface Plasmon Resonance Biosensor for the DNA Hybridization Detection: Simulation Analysis. Optics Communications, 383, 186-190. https://doi.org/10.1016/j.optcom.2016.09.015

[3] Hossain, M.B. and Rana, M. (2016) Graphene Coated High Sensitive Surface Plasmon Resonance Biosensor for Sensing DNA Hybridization. Sensor Letters, 14, 145-152. https://doi.org/10.1166/sl.2016.3596

[4] Hossain, M.B. and Rana, M.M. (2016) DNA Hybridization Detection Based on Resonance Frequency Readout in Graphene on Au SPR Biosensor. Journal of Sensors, 2016, Article ID: 6070742. https://doi.org/10.1155/2016/6070742

[5] Rahman, M.S., Anower, M.S., Hasan, M.R., Hossain, M.B. and Haque, M.I. (2017) Design and Numerical Analysis of Highly Sensitive Au- $\mathrm{MoS}_{2}$-Graphene Based Hybrid Surface Plasmon Resonance Biosensor. Optics Communications, 396, 36-43. https://doi.org/10.1016/j.optcom.2017.03.035

[6] Rahman, M.S., Anower, M.S., Rahman, M.K., Hasan, M.R., Hossain, M.B., et al. (2017) Modeling of a Highly Sensitive $\mathrm{MoS}_{2}$-Graphene Hybrid Based Fiber Optic SPR Biosensor for Sensing DNA Hybridization. International Journal for Light and 
Electron Optics, 140, 989-997. https://doi.org/10.1016/j.ijleo.2017.05.001

[7] Rahman, M.S., Hossain, M.B. and Rana, M.M. (2016) Sensitivity Enhancement of Porous Silicon Based SPR Sensor Using Graphene-MoS2 Hybrid Structure. 2nd International Conference on Electrical, Computer \& Telecommunication Engineering, Rajshahi, December 2016, 1-4. https://doi.org/10.1109/ICECTE.2016.7879607

[8] Hossain, Md.B., Khan, M.M.R., Rahman, Md.S., Badrudduza, S.S.B., Sabiha, M.M. and Rana, Md.M. (2019) Graphene- $\mathrm{MoS}_{2}-\mathrm{Au}-\mathrm{TiO}_{2}-\mathrm{SiO}_{2}$ Hybrid SPR Biosensor: A New Window for Formalin Detection. Journal of Materials and Applications, 8. https://doi.org/10.32732/jma.2019.8.2.51

[9] Kretchmann, E. and Raether, Z.H. (1968) Radiative Decay of Non-Radiative Surface Plasmons Excited by Light. Zeitschrift für Naturforschung A, 23, 2135-2136.

https://doi.org/10.1515/zna-1968-1247

[10] Russel, P. (2003) Photonic Crystal Fibers. Science, 299, 358-362. https://doi.org/10.1126/science.1079280

[11] Rifat, A.A., Amouzad Mahdiraji, G., Shee, Y.G., Jubayer Shawon, M. and Mahamd Adikan, F.R. (2016) A Novel Photonic Crystal Fiber Biosensor Using Surface Plasmon Resonance. Procedia Engineering, 140, 1-7. https://doi.org/10.1016/j.proeng.2015.08.1107

[12] Jorgenson, R. and Yee, S. (1993) A Fiber-Optic Chemical Sensor Based on Surface Plasmon Resonance. Sensors and Actuators B: Chemical, 12, 213-220. https://doi.org/10.1016/0925-4005(93)80021-3

[13] Dash, J.N. and Jha, R. (2014) SPR Biosensor Based on Polymer PCF Coated with Conducting Metal Oxide. IEEE Photonics Technology Letters, 26, 595-598. https://doi.org/10.1109/LPT.2014.2301153

[14] Jha, R. and Dash, J. (2014) Graphene Based Birefringent Photonic Crystal Fiber Sensor Using Surface Plasmon Resonance. IEEE Photonics Technology Letters, 26, 1092-1095. https://doi.org/10.1109/LPT.2014.2315233

[15] Hossain, M.B., Rana, M.M., Abdulrazak, L.F., Mitra, S. and Rahman, M. (2019) Design and Analysis of Graphene-MoS2 Hybrid Layer Based SPR Biosensor with $\mathrm{TiO}_{2}-\mathrm{SiO}_{2}$ Nano Film for Formalin Detection: Numerical Approach. Optical and Quantum Electronics, 51, 195-207. https://doi.org/10.1007/s11082-019-1911-Z

[16] Otupiri, R., Akowuah, E., Haxha, S., Ademgil, H., AbdelMalek, F. and Aggoun, A. (2014) A Novel Birefrigent Photonic Crystal Fibre Surface Plasmon Resonance Biosensor. IEEE Photonics Journal, 6, Article ID: 6801711. https://doi.org/10.1109/JPHOT.2014.2335716

[17] Hossain, M.B., Rana, M.M., Abdulrazak, L.F. and Mitra, S. (2019) Graphene-MoS with $\mathrm{TiO}_{2}-\mathrm{SiO}_{2}$ Layers Based Surface Plasmon Resonance Biosensor: Numerical Development for Formalin Detection. Biochemistry and Biophysics Reports, 18, Article ID: 100639. https://doi.org/10.1016/j.bbrep.2019.100639

[18] Rifat, A., Mahdiraji, G., Chow, D., Shee, Y., Ahmed, R. and Adikan, F. (2015) Photonic Crystal Fiber-Based Surface Plasmon Resonance Sensor with Selective Analyte Channels and Graphene-Silver Deposited Core. Sensors, 15, 11499-11510. https://doi.org/10.3390/s150511499

[19] Kravets, V., Jalil, R., Kim, Y.-J., Ansell, D., Aznakayeva, D., Thackray, B., et al. (2014) Graphene-Protected Copper and Silver Plasmonics. Scientific Reports, 4, Article No. 5517. https://doi.org/10.1038/srep05517

[20] Hossain, M.B., Akib, T.B.A., Abdulrazak, L.F. and Rana, Md.M. (2019) Numerical Modeling of Graphene-Coated Fiber Optic Surface Plasmon Resonance Biosensor for BRCA 1 and BRCA 2 Genetic Breast Cancer Detection. Optical Engineering, 58, 
Article ID: 037104. https://doi.org/10.1117/1.OE.58.3.037104

[21] Islam, M.M., Islam, Md.M, Shimul, Y.C., Rahman, A., Ruhe, A.A., Hassan, M., et al. (2019) FDTD Analysis Fiber Optic SPR Biosensor for DNA Hybridization: A Numerical Demonstration with Graphene. Journal of Materials and Applications, 8, 13-19. https://doi.org/10.32732/jma.2019.8.1.13

[22] Aoni, R.A., Ahmed, R., Alam, M.M. and Razzak, S.A. (2013) Optimum Design of a Nearly Zero Ultra-Flattened Dispersion with Lower Confinement Loss Photonic Crystal Fibers for Communication Systems. International Journal of Scientific and Engineering Research, 4, 1-4.

[23] Hossain, M.B., Islam, M.M., Abdulrazak, L.F., Rana, M.M., Akib, T.B.A. and Hassan, M. (2019) Graphene-Coated Optical Fiber SPR Biosensor for BRCA1 and BRCA2 Breast Cancer Biomarker Detection: A Numerical Design-Based Analysis. Photonic Sensors, 9, 1-13. https://doi.org/10.1007/s13320-019-0556-7

[24] Gauvreau, B., Hassani, A., Fassi Fehri, M., Kabashin, A. and Skorobogatiy, M.A. (2007) Photonic Bandgap Fiber-Based Surface Plasmon Resonance Sensors. Optics Express, 15, 11413-11426. https://doi.org/10.1364/OE.15.011413

[25] Hossain, M.B., Hassan, M., Abdulrazak, L.F., Rana, M.M., Islam, M.M. and Rahman, M.S. (2019) Graphene-MoS2-Au- $\mathrm{TiO}_{2}-\mathrm{SiO}_{2}$ Hybrid SPR Biosensor for Formalin Detection: Numerical Analysis and Development. Advanced Materials Letters, 10, 656-662.

[26] Habib, M.A., Anower, M.S., Abdulrazak, L.F. and Reza, M.S. (2019) Hollow Core Photonic Crystal Fiber for Chemical Identification in Terahertz Regime. Plasmonics, 52, Article ID: 101933. https://doi.org/10.1016/j.yofte.2019.101933

[27] Rahman, M.S., Anower, M.S. and Abdulrazak, L.F. (2019) Utilization of a Phosphorene-Graphene/TMDC Heterostructure in a Surface Plasmon Resonance-Based Fiber Optic Biosensor. Photonics and Nanostructures-Fundamentals and Applications, 35, Article ID: 100711. https://doi.org/10.1016/j.photonics.2019.100711

[28] Rahman, M.S., Anower, M.S., Abdulrazak, L.F. and Rahman, M.M. (2019) Modeling of a Fiber-Optic Surface Plasmon Resonance Biosensor Employing Phosphorene for Sensing Applications. Optical Engineering, 58, Article ID: 037103. https://doi.org/10.1117/1.OE.58.3.037103

[29] Habib, M.A., Reza, M.S., Abdulrazak, L.F. and Anower, M.S. (2019) Extremely High Birefringent and Low Loss Microstructure Optical Waveguide: Design and Analysis. Optics Communications, 446, 93-99. https://doi.org/10.1016/j.optcom.2019.04.060

[30] Rahman, M.S., Noor, S.S., Anower, M.S., Abdulrazak, L.F., Rahman, M.M. and Rikta, K.A. (2019) Design and Numerical Analysis of a Graphene-Coated Fiber-Optic SPR Biosensor Using Tungsten Disulfide. Photonics and Nanostructures-Fundamentals and Applications, 33, 29-35. https://doi.org/10.1016/j.photonics.2018.11.005

[31] M. Saifur Rahman, Md. Shamim Anower, Lway Faisal Abdulrazak. (2019) Modeling of a Fiber Optic SPR Biosensor Employing Tin Selenide (SnSe) Allotropes. Results in Physics, 15, Article ID: 102623. https://doi.org/10.1016/j.rinp.2019.102623

[32] Hossain, B., Tasnim, T., Abdulrazak, L.F., Rana, M. and Islam, R. (2019) A Numerical Approach to Design the Kretschmann Configuration Based Refractive Index Graphene- $\mathrm{MoS}_{2}$ Hybrid Layers with $\mathrm{TiO}_{2}-\mathrm{SiO}_{2}$ Nano for Formalin Detection. Photonic Sensors, 1-13. https://doi.org/10.1007/s13320-019-0566-5 